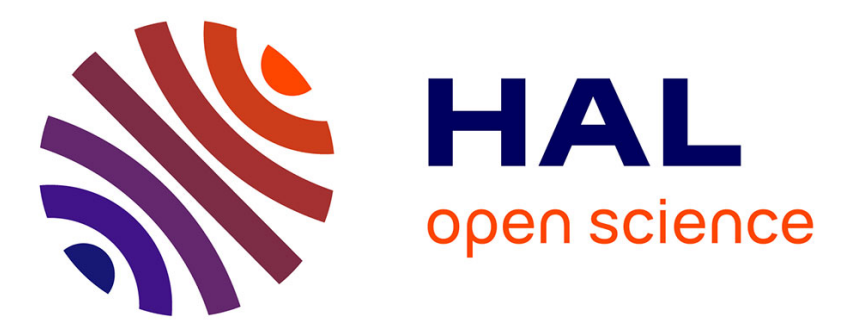

\title{
Preliminary Investigation of a Nonconforming Discontinuous Galerkin Method for Solving the Time-Domain Maxwell Equations
}

Hassan Fahs, Loula Fatima Fezoui, Stéphane Lanteri, Francesca Rapetti

\section{To cite this version:}

Hassan Fahs, Loula Fatima Fezoui, Stéphane Lanteri, Francesca Rapetti. Preliminary Investigation of a Nonconforming Discontinuous Galerkin Method for Solving the Time-Domain Maxwell Equations. IEEE Transactions on Magnetics, 2008, 44 (6), pp.1254-1257. 10.1109/TMAG.2007.916577 . hal00664104

\section{HAL Id: hal-00664104 https://hal.science/hal-00664104}

Submitted on 9 Jul 2013

HAL is a multi-disciplinary open access archive for the deposit and dissemination of scientific research documents, whether they are published or not. The documents may come from teaching and research institutions in France or abroad, or from public or private research centers.
L'archive ouverte pluridisciplinaire HAL, est destinée au dépôt et à la diffusion de documents scientifiques de niveau recherche, publiés ou non, émanant des établissements d'enseignement et de recherche français ou étrangers, des laboratoires publics ou privés. 


\title{
Preliminary investigation of a non-conforming discontinuous Galerkin method for solving the time-domain Maxwell equations
}

\author{
Hassan Fahs, Loula Fezoui, Stéphane Lanteri and Francesca Rapetti
}

\begin{abstract}
This paper is concerned with the design of a high order discontinuous Galerkin (DG) method for solving the 2D time-domain Maxwell equations on non-conforming triangular meshes. The proposed DG method allows for using nonconforming meshes with arbitrary-level hanging nodes. This method combines a centered approximation for the evaluation of fluxes at the interface between neighboring elements of the mesh, with a leap-frog time integration scheme. Numerical experiments are presented which both validate the theoretical results and provide further insights regarding to the practical performance of the proposed DG method, particulary when non-conforming meshes are employed.
\end{abstract}

Index Terms-Maxwell's equations, discontinuous Galerkin method, non-conforming triangular meshes.

\section{INTRODUCTION}

A LOT of methods have been developed for the numerical solution of the time-domain Maxwell equations. Finite difference time-domain (FDTD) methods based on Yee's scheme [1] (a time explicit method defined on a staggered mesh) are still prominent because of their simplicity and their non-dissipative nature (they hold an energy conservation property which is an important ingredient in the numerical simulation of unsteady wave propagation problems). Unfortunately, the discretization of objects with complex shapes or small geometrical details using cartesian meshes hardly yields an efficient numerical methodology. A natural approach to overcome this difficulty in the context of the FDTD method is to resort to non-conforming local refinement. However, instabilities have often been reported for these methods and rarely studied theoretically until very recently [2].

Finite element methods can handle unstructured meshes and complex geometries. However, the development of high-order versions of such methods for solving Maxwell's equations has been relatively slow. A primary reason is the appearance of spurious, non-physical solutions when a straightforward nodal continuous Galerkin finite element scheme is used to approximate the Maxwell curl-curl equations. Bossavit made the fundamental observation that the use of special curlconforming elements [3] would overcome the probleme of spurious modes by mimicking properties of vector algebra [4]. Although very successful, such approximations are not

H. Fahs, L. Fezoui and S. Lanteri are with INRIA, 06902 Sophia Antipolis, France (e-mail: $\{$ Hassan.Fahs, Loula.Fezoui, Stephane.Lanteri\}@inria.fr).

F. Rapetti is with the Nice/Sophia Antipolis University, J.A. Dieudonné Mathematics Lab., UMR CNRS 6621, 06108 Nice, France (e-mail: Francesca.Rapetti@unice.fr).

Manuscript received June 24, 2007. entirely void of problems: the algebraic problems are larger than for nodal elements and the conformity requirements of the continuous Galerkin formulation makes adaptivity complex.

In an attempt to offer an alternative to the classical finite element formulation based on edge elements, we consider here discontinuous Galerkin formulations [5] based on high order nodal elements for solving the first order time-domain Maxwell's equations. Discontinuous Galerkin time-domain (DGTD) methods can handle unstructured meshes, deal with discontinuous coefficients and solutions, by locally varying polynomial order, and get rid of differential operators (and finite element mass matrices) by using Green's formula for the integration over control volumes. People rediscover indeed the abilities of these methods to handle complicated geometries, media and meshes, to achieve high order accuracy by simply choosing suitable basis functions, to allow long-range time integrations and, last but not least, to remain highly parallelizable at the end. Whereas high order discontinous Galerkin timedomain methods have been developed on hexahedral [6] and tetrahedral [7] meshes, the design of non-conforming discontinuous Galerkin time-domain methods is still in its infancy. In practice, the non-conformity can result from a local refinement of the mesh (i.e. $h$-refinement), of the interpolation order (i.e. $p$-enrichment) or of both of them (i.e. $h p$-refinement).

The present study is a preliminary step towards the development of a non-conforming discontinuous Galerkin method for solving the three-dimensional time-domain Maxwell equations on unstructured tetrahedral meshes. Here, we consider the two-dimensional case and we concentrate on the situation where the discretization is locally refined in a non-conforming way yielding triangular meshes with arbitrary-level hanging nodes. In this context, the contributions of this work are on the one hand, a theoretical and numerical stability analysis of high order DGTD methods on non-conforming triangular meshes and, on the other hand, a numerical assessment of the convergence of such DGTD methods.

\section{Discontinuous Galerkin Time-Domain method}

We consider the two-dimensional Maxwell equations in the $\mathrm{TM} z$ polarization on a bounded domain $\Omega \subset \mathbb{R}^{2}$ :

$$
\left\{\begin{array}{l}
\epsilon \frac{\partial E_{z}}{\partial t}-\frac{\partial H_{y}}{\partial x}+\frac{\partial H_{x}}{\partial y}=0 \\
\mu \frac{\partial H_{x}}{\partial t}+\frac{\partial E_{z}}{\partial y}=0, \text { and } \mu \frac{\partial H_{y}}{\partial t}-\frac{\partial E_{z}}{\partial x}=0
\end{array}\right.
$$


where the unknowns are $\mathbf{E}=\left(0,0, E_{z}\right)$ and $\mathbf{H}=\left(H_{x}, H_{y}, 0\right)$, the electric and magnetic fields, respectively. The electric permittivity $\epsilon$ and the magnetic permeability $\mu$ of the medium are assumed to be piecewise constant. We assume that the field components as well as the material parameters $\epsilon$ and $\mu$ do not depend on the $z$ coordinate. The boundary $\partial \Omega$ is assumed to be a perfect electric conductor.

We consider a partition $\mathcal{T}_{h}$ of $\Omega$ into a set of triangles $T_{i}$ of size $h_{i}$ such that the mesh size $h=\max _{T_{i} \in \mathcal{T}_{h}} h_{i}$. To each $T_{i} \in \mathcal{T}_{h}$ we assign an integer $p_{i}>0$ and we collect the $p_{i}$ in the vector $p=\left\{p_{i}: T_{i} \in \mathcal{T}_{h}\right\}$. Within this construction we admit meshes with possibly hanging nodes, i.e., non-conforming meshes where triangle vertices can lie in the interior of edges of other triangles. Each triangle $T_{i}$ is assumed to be the image, under a smooth bijective and affine mapping of a fixed master triangle $\hat{T}=\{\hat{x}, \hat{y} \mid \hat{x}, \hat{y} \geq 0 ; \hat{x}+\hat{y} \leq 1\}$. In the following, we seek for approximate solutions to (1) in the finite dimensional space $V_{p}\left(\mathcal{T}_{h}\right):=\left\{v \in L^{2}(\Omega): v_{\mid T_{i}} \in \mathbb{P}_{p_{i}}\left(T_{i}\right), \forall T_{i} \in \mathcal{T}_{h}\right\}$, where $\mathbb{P}_{p_{i}}\left(T_{i}\right)$ denotes the space of polynomials $\left\{\varphi_{i j}\right\}_{j=1}^{d_{i}}$ of total degree at most $p_{i}$ on the element $T_{i}$. The space $V_{p}\left(\mathcal{T}_{h}\right)$ has the dimension $d_{i}$, the local number of degrees of freedom (dof). Note that the polynomial degree $p_{i}$ may vary from element to element in the mesh and that a function $v_{h}^{p} \in V_{p}\left(\mathcal{T}_{h}\right)$ is discontinuous across element interfaces. For two distinct triangles $T_{i}$ and $T_{k}$ in $\mathcal{T}_{h}$, the intersection $T_{i} \cap T_{k}$ is an (oriented) edge $s_{i k}$ which we will call interface, with oriented normal vector $\vec{n}_{i k}$. For the boundary interfaces, the index $k$ corresponds to a fictitious element outside the domain. By non-conforming interface we mean an interface $s_{i k}$ which has at least one of its two vertices in a hanging node or such that $p_{\left.i\right|_{s_{i k}}} \neq p_{\left.k\right|_{s_{i k}}}$ or both of them. Finally, we denote by $\mathcal{V}_{i}$ the set of indices of the elements neighboring $T_{i}$.

The DGTD method at the heart of this study is based on a leap-frog time scheme ( $E_{z}$ is computed at integer time-stations and $H_{x}$ and $H_{y}$ at half-integer time-stations) and totally centered numerical fluxes at the interface between elements. Decomposing $H_{x}, H_{y}$ and $E_{z}$ on element $T_{i}$ according to:

$$
H_{\mathbf{x}}\left(., t^{n+\frac{1}{2}}\right)=\sum_{j=1}^{d_{i}} H_{\mathbf{x}_{i j}}^{n+\frac{1}{2}} \varphi_{i j}, \quad E_{z}\left(., t^{n}\right)=\sum_{j=1}^{d_{i}} E_{z_{i j}}^{n} \varphi_{i j},
$$

where $\mathbf{x} \in\{x, y\}$. Using the notations $\mathbf{E}_{z_{i}}^{n}=$ $\left(E_{z_{i 1}}^{n}, \ldots, E_{z_{i d_{i}}}^{n}\right)^{t}$ and $\mathbf{H}_{\mathbf{x}_{i}}^{n+\frac{1}{2}}=\left(H_{\mathbf{x}_{i 1}}^{n+\frac{1}{2}}, \ldots, H_{\mathbf{x}_{i d_{i}}}^{n+\frac{1}{2}}\right)^{t}$, the DGTD- $\mathbb{P}_{p_{i}}$ method writes:

$$
\left\{\begin{aligned}
\epsilon_{i} \mathbb{M}_{i} \frac{\mathbf{E}_{z_{i}}^{n+1}-\mathbf{E}_{z_{i}}^{n}}{\Delta t}= & -\mathbb{K}_{i}^{x} \mathbf{H}_{y_{i}}^{n+\frac{1}{2}}+\mathbb{K}_{i}^{y} \mathbf{H}_{x_{i}}^{n+\frac{1}{2}} \\
& +\sum_{k \in \mathcal{V}_{i}}\left(\mathbb{G}_{x_{i k}}^{n+\frac{1}{2}}-\mathbb{G}_{y_{i k}}^{n+\frac{1}{2}}\right), \\
\mu_{i} \mathbb{M}_{i} \frac{\mathbf{H}_{x_{i}}^{n+\frac{1}{2}}-\mathbf{H}_{x_{i}}^{n-\frac{1}{2}}}{\Delta t}= & \mathbb{K}_{i}^{y} \mathbf{E}_{z_{i}}^{n}-\sum_{k \in \mathcal{V}_{i}} \mathbb{F}_{y_{i k}}^{n}, \\
\mu_{i} \mathbb{M}_{i} \frac{\mathbf{H}_{y_{i}}^{n+\frac{1}{2}}-\mathbf{H}_{y_{i}}^{n-\frac{1}{2}}}{\Delta t}= & -\mathbb{K}_{i}^{x} \mathbf{E}_{z_{i}}^{n}+\sum_{k \in \mathcal{V}_{i}} \mathbb{F}_{x_{i k}}^{n},
\end{aligned}\right.
$$

where $\mathbb{M}_{i}$ is the local mass (symmetric positive definite) matrix, and $\mathbb{K}_{i}^{\mathbf{x}}$ is the (skew-symmetric) stiffness matrix. The vector quantities $\mathbb{F}_{\mathbf{x}_{i k}}^{n}$ and $\mathbb{G}_{\mathbf{x}_{i k}}^{n+\frac{1}{2}}$ are defined as:

$$
\mathbb{F}_{\mathbf{x}_{i k}}^{n}=\mathbb{S}_{i k}^{\mathbf{x}} \mathbf{E}_{z_{k}}^{n}, \quad \mathbb{G}_{\mathbf{x}_{i k}}^{n+\frac{1}{2}}=\mathbb{S}_{i k}^{\mathbf{x}} \mathbf{H}_{\mathbf{x}_{k}}^{n+\frac{1}{2}},
$$

where $\mathbb{S}_{i k}^{\mathbf{x}}$ is the $d_{i} \times d_{k}$ interface matrix on $s_{i k}$ which verifies ${ }^{t} \mathbb{S}_{i k}^{\mathbf{x}}=-\mathbb{S}_{k i}^{\mathbf{x}}$ (if $s_{i k}$ is an internal interface) and ${ }^{t} \mathbb{S}_{i k}^{\mathbf{x}}=\mathbb{S}_{i k}^{\mathbf{x}}$ (if $s_{i k}$ is a boundary interface). Note that, for non-conforming interfaces, we calculate the matrix $\mathbb{S}_{i k}^{\mathrm{x}}$ by using a Gaussian quadrature formula [8].

In [8], a numerical dispersion has been observed when a low order conforming DGTD- $\mathbb{P}_{p}\left(p=0,1\right.$ and $p_{i}=p$ everywhere) is applied. This dispersion error is not reduced notably by using a $h$-refinement strategy (i.e. modifying $h$ for a fixed $p$, yielding non-conforming locally refined meshes). On the other hand, the dispersion error is minimized when a $p$-enrichment strategy (i.e. modifying $p$ for a fixed $h$ ) is used. However, the latter approach requires a large number of dof thus increases substantially the computing time and memory usage. We propose here a $h p$-like DGTD method where we combine $h$-refinement and $p$-enrichment strategies. This method consists in using a high polynomial order in the coarse (i.e. not refined) mesh and a low order one in the refined region. The resulting scheme is referred to as a DGTD- $\mathbb{P}_{p_{c}}: \mathbb{P}_{p_{f}}$ method where $p_{c}$ and $p_{f}$ are the polynomial degrees in the coarse and fine elements respectively. This kind of scheme is a first step towards a fully adaptive $h p$-refinement method relying on appropriate error estimators.

\section{STABILITY ANALYSIS}

On any non-conforming mesh, the DGTD method exactly conserves the following energy [8]:

$$
\begin{aligned}
\mathcal{E}^{n}=\frac{1}{2} \sum_{i}\left(\epsilon_{i}{ }^{t} \mathbf{E}_{z_{i}}^{n} \mathbb{M}_{i} \mathbf{E}_{z_{i}}^{n}\right. & +\mu_{i}{ }^{t} \mathbf{H}_{x_{i}}^{n-\frac{1}{2}} \mathbb{M}_{i} \mathbf{H}_{x_{i}}^{n+\frac{1}{2}} \\
& \left.+\mu_{i}{ }^{t} \mathbf{H}_{y_{i}}^{n-\frac{1}{2}} \mathbb{M}_{i} \mathbf{H}_{y_{i}}^{n+\frac{1}{2}}\right),
\end{aligned}
$$

and one can show that $\mathcal{E}^{n}$ is a positive definite quadratic form of all numerical unknowns under the CFL-like sufficient stability condition on the time step $\Delta t$ :

$$
\forall i, \forall k \in \mathcal{V}_{i}, \quad c_{i} \Delta t\left(2 \alpha_{i}+\beta_{i k}\right) \leq 4 \min \left(\frac{\left|T_{i}\right|}{P_{i}^{x}}, \frac{\left|T_{i}\right|}{P_{i}^{y}}\right),
$$

where $c_{i}$ is the local speed of propagation, $\left|T_{i}\right|$ is the surface of $T_{i}$ and $P_{i}^{\mathbf{x}}=\sum_{k \in \mathcal{V}_{i}}\left|n_{i k \mathbf{x}}\right|$. The constants $\alpha_{i}$ and $\beta_{i k}\left(k \in \mathcal{V}_{i}\right)$ verify some inequalities [8] on $T_{i}$ and $s_{i k}$ :

$$
\left\{\begin{array}{l}
\forall \xi_{i} \in \operatorname{Span}\left\{\varphi_{i j}, 1 \leq j \leq d_{i}\right\}, \mathbf{x} \in\{x, y\} \\
\left\|\frac{\partial \xi_{i}}{\partial \mathbf{x}}\right\|_{T_{i}} \leq \frac{\alpha_{i} P_{i}^{\mathbf{x}}}{\left|T_{i}\right|}\left\|\xi_{i}\right\|_{T_{i}}, \quad\left\|\xi_{i}\right\|_{s_{i k}}^{2} \leq \beta_{i k} \frac{\left\|\vec{n}_{i k}\right\|}{\left|T_{i}\right|}\left\|\xi_{i}\right\|_{T_{i}}^{2} .
\end{array}\right.
$$

The values of $\alpha_{i}$ only depend on the local polynomial order $p_{i}$ while the values of $\beta_{i k}$ depend on $p_{i}$ and on the number of hanging nodes in the interface $s_{i k}$. Consequently, if $p_{i}$ and the number of hanging nodes increase, the theoretical CFL values become restrictive [8]. We report here on the CFL values evaluated numerically (i.e. by assessing the limit beyond which we observe a growth of the discrete energy). The corresponding values of $\mathrm{CFL}_{p_{i}}^{\text {num }}$ are summarized in Tab. I and II for the DGTD- $\mathbb{P}_{p_{c}}: \mathbb{P}_{p_{f}}$ and DGTD- $\mathbb{P}_{p}$ methods respectively. 
One can note that for $p_{c}=p_{f}+1=p+1$, the DGTD- $\mathbb{P}_{p_{c}}: \mathbb{P}_{p_{f}}$ method has the same stability limit as the DGTD- $\mathbb{P}_{p}$ scheme, as long as the mesh is actually refined. This is not a surprise, since the DGTD- $\mathbb{P}_{p_{c}}$ scheme, which has a reduced stability domain, is only used on elements of the coarse mesh (which are at least twice larger than elements of the refined mesh).

TABLE I

NUMERICAL CFL OF THE DGTD- $\mathbb{P}_{p}$ METHOD.

\begin{tabular}{c|c|c|c|c|c}
\hline$p_{c}=p_{f}=p$ & $\mathbf{0}$ & $\mathbf{1}$ & $\mathbf{2}$ & $\mathbf{3}$ & $\mathbf{4}$ \\
\hline \hline $\mathrm{CFL}_{p}^{\text {num }}$ & 1.0 & 0.3 & 0.2 & 0.15 & 0.1 \\
\hline
\end{tabular}

TABLE II

NUMERICAL CFL OF THE DGTD- $\mathbb{P}_{p_{c}}: \mathbb{P}_{p_{f}}$ METHOD.

\begin{tabular}{c|c|c|c|c|c|c}
\hline$p_{c}: p_{f}$ & $\mathbf{1 : 0}$ & $\mathbf{2 : 0}$ & $\mathbf{2 : 1}$ & $\mathbf{3 : 1}$ & $\mathbf{3 : 2}$ & $\mathbf{4 : 2}$ \\
\hline \hline $\mathrm{CFL}_{p_{c}: p_{f}}^{\text {num }}$ & 1.0 & 0.4 & 0.3 & 0.25 & 0.15 & 0.15 \\
\hline
\end{tabular}

\section{CONVERgence ANALYSis}

In [7] it is shown that the convergence order of the centered in space and time DGTD- $\mathbb{P}_{p}$ method, in the case of conforming simplicial meshes, is:

$$
\mathcal{O}\left(T h^{\min (s, p)}\right)+\mathcal{O}\left(\Delta t^{2}\right)
$$

where $\Delta t$ is the time step over the interval $[0, T]$ and the solution belongs to $H^{s}(\Omega)$ with $s>1 / 2$. Our attention is turned in the validity of this result in the case of nonconforming meshes using the DGTD- $\mathbb{P}_{p}$ and DGTD- $\mathbb{P}_{p_{c}}: \mathbb{P}_{p_{f}}$ methods, and a preliminary answer is given here on the basis of numerical simulations.

\section{A. Eigenmode in a PEC cavity filled with vaccum}

The first test case that we consider is the propagation of an eigenmode in a unitary PEC cavity with $\epsilon=\mu=1$ in normalized units. Numerical simulations make use of triangular meshes of the square $[0,1] \times[0,1]$ and non-conforming meshes are obtained thanks to local refinements of a rectangular zone as shown on Fig. 2 right. Fig. 1 and 2 illustrate respectively the numerical convergence of the DGTD- $\mathbb{P}_{p}$ and DGTD$\mathbb{P}_{p_{c}}: \mathbb{P}_{p_{f}}$ methods using conforming and non-conforming triangular meshes, in terms of the evolution of the $L^{2}$-error as a function of the square root of the number of dof. These errors are measured after two periods. Corresponding asymptotic convergence orders are summarized in Tab. III and IV. As it could be expected from the use of a second order accurate time integration scheme, the asymptotic convergence order is bounded above by 2 independently of the interpolation order $p$ (excepted for $p=2=p_{f}$ on non-conforming mesh for which we obtain convergence orders higher than 2) and higher order convergence rates will require more accurate time integration schemes. Furthermore, we can observe that for $p=0,1$, the convergence order is $\mathcal{O}\left(h^{p+1}\right)$. From these points of view, it seems that the formula (2) is sub-optimal and suggests that theoretical convergence study conducted in [7] might be improved in view of the development of $h$ - and $h p$-adaptive
DGTD methods. Moreover, we have observed in the case of DGTD- $\mathbb{P}_{p_{c}}: \mathbb{P}_{p_{f}}$ method that it is not necessary to increase $p_{c}$ to more than $p_{f}+2$, since the convergence order is not improved.
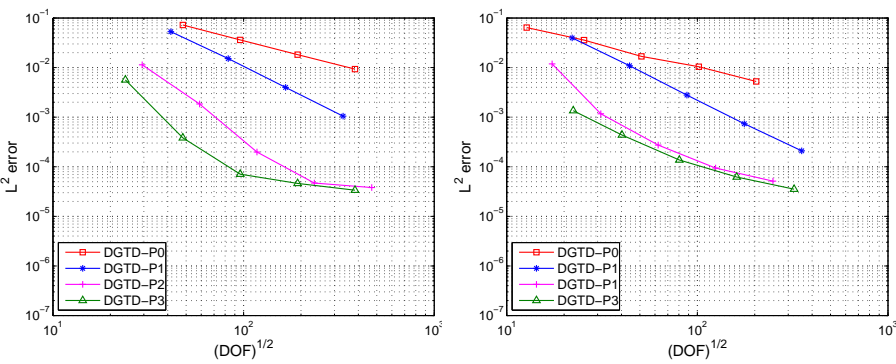

Fig. 1. Numerical convergence of the DGTD- $\mathbb{P}_{p}$ method with $h$-refinement. Non-Conforming (left) and conforming (right) triangular mesh.
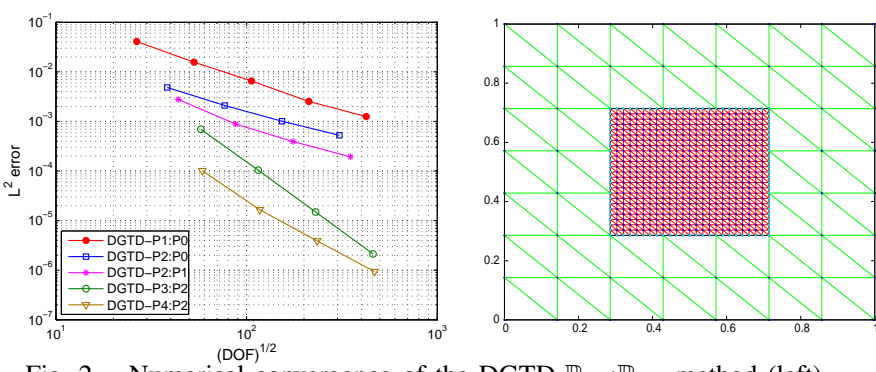

Fig. 2. Numerical convergence of the DGTD- $\mathbb{P}_{p_{c}}: \mathbb{P}_{p_{f}}$ method (left) on the non-conforming triangular mesh (right).

TABLE III

NUMERICAL CONVERGENCE OF THE DGTD- $\mathbb{P}_{p}$ METHOD.

Conforming triangular mesh: convergence order.

\begin{tabular}{c|c|c|c|c}
\hline Interpolation order $p$ & $\mathbf{0}$ & $\mathbf{1}$ & $\mathbf{2}$ & $\mathbf{3}$ \\
\hline \hline Convergence order & 0.90 & 1.90 & 1.98 & 1.37 \\
\hline
\end{tabular}

Non-conforming triangular mesh: convergence order.

\begin{tabular}{c|c|c|c|c}
\hline Interpolation order $p$ & $\mathbf{0}$ & $\mathbf{1}$ & $\mathbf{2}$ & $\mathbf{3}$ \\
\hline \hline Convergence order & 0.99 & 1.89 & 2.17 & 1.78 \\
\hline
\end{tabular}

TABLE IV

Numerical CONVERGENCE OF THE DGTD- $\mathbb{P}_{p_{c}}: \mathbb{P}_{p_{f}}$ METHOD.

Non-conforming triangular mesh: convergence order.

\begin{tabular}{c|c|c|c|c|c}
\hline Interpolation orders $p_{c}: p_{f}$ & $\mathbf{1 : 0}$ & $\mathbf{2 : 0}$ & $\mathbf{2 : 1}$ & $\mathbf{3 : 2}$ & $\mathbf{4 : 2}$ \\
\hline \hline Convergence order & 1.27 & 1.07 & 1.27 & 2.78 & 2.24 \\
\hline
\end{tabular}

Tab. V shows the CPU times, the $L^{2}$ errors, the number of time steps (\#tt) and the number of dof (\# dof) for some cases of the proposed methods. One can see that to achieve a given accuracy the gains of CPU time and memory consumption is notable if we use the DGTD- $\mathbb{P}_{p_{c}}: \mathbb{P}_{p_{f}}$ method. Moreover, to reach a high accuracy, the cost of the non-conforming DGTD$\mathbb{P}_{p}$ method is comparable with the conforming one.

\section{B. Eigenmode in a PEC cavity with a dielectric material}

In this problem, a lossless dielectric with a relative permittivity $\epsilon_{2}$ is enclosed by air in the $x$ direction, and the media 
TABLE V

$L^{2}$ ERROR, CPU TIME (SECONDS), NUMBER OF DOF AND NUMBER OF TIME STEPS MEASURED AFTER TWO PERIODS.

DGTD- $\mathbb{P}_{p}:$ Conforming triangular mesh.

\begin{tabular}{c|c|c|c}
\hline$p$ & $\mathbf{1}$ & $\mathbf{2}$ & $\mathbf{3}$ \\
\hline$L^{2}$ error, CPU & $2.78 \mathrm{E}-03, \quad 10$ & $5.13 \mathrm{E}-05, \quad 324$ & $3.53 \mathrm{E}-05, \quad 658$ \\
$\#$ dof, \# $\Delta t$ & 7740,600 & $61500, \quad 4800$ & $102400, \quad 7200$ \\
\hline
\end{tabular}

DGTD- $\mathbb{P}_{p}:$ Non-conforming triangular mesh.

\begin{tabular}{c|c|c|c}
\hline$p$ & $\mathbf{1}$ & $\mathbf{2}$ & $\mathbf{3}$ \\
\hline$L^{2}$ error, CPU & $3.98 \mathrm{E}-03, \quad 154$ & $4.69 \mathrm{E}-05, \quad 580$ & $4.61 \mathrm{E}-05, \quad 489$ \\
$\#$ dof, \# $\Delta t$ & $27552, \quad 3200$ & $55224, \quad 6400$ & $36860, \quad 6400$ \\
\hline
\end{tabular}

\begin{tabular}{c|c|c|c}
\multicolumn{4}{c}{ DGTD- $\mathbb{P}_{p_{c}}: \mathbb{P}_{p_{f}}:$ Non-conforming triangular mesh. } \\
\hline$p_{c}: p_{f}$ & $\mathbf{2 : 1}$ & $\mathbf{3 : 2}$ & $\mathbf{4 : 2}$ \\
\hline$L^{2}$ error, CPU & $2.78 \mathrm{E}-03,2$ & $4.56 \mathrm{E}-05, \quad 79$ & $1.66 \mathrm{E}-05, \quad 51$ \\
$\#$ dof, \# $\Delta t$ & 1935,625 & 24024,2156 & 13690,2400 \\
\hline
\end{tabular}

are nonmagnetic and homogeneous along the $y$ direction. The computational domain $\Omega=\{(x, y),|x| \leq 1,|y| \leq 1\}$ is enveloped by PEC walls. The permittivity is given as $\epsilon=\epsilon_{1}$ if $\frac{1}{2} \leq|x| \leq 1$ and $|y| \leq 1$, and $\epsilon=\epsilon_{2}$ if $|x| \leq \frac{1}{2}$ and $|y| \leq 1$, where $\epsilon_{1}=1$ and $\epsilon_{2}=2.25$. An analytical solution for time varying electromagnetic fields is known for this problem [9]. Contour lines of the $H_{y}$ and $E_{z}$ components at time $t=1.0$ are shown in Fig. 3. The non-conforming meshes are obtained by refining the heterogeneous zone. Numerical convergence orders measured at time $t=0.65$ are summarized in Tab. VI and VII. One can note that the convergence of the DGTD$\mathbb{P}_{p}(p \geq 2)$ method is notably slower than what was observed with the previous test case, which is the result of the presence of a material interface in the domain. On the other hand, Tab. VII shows that the convergence rate is improved with the DGTD- $\mathbb{P}_{p_{c}}: \mathbb{P}_{p_{f}}$ method using a low interpolation order in the heterogenuous zone.
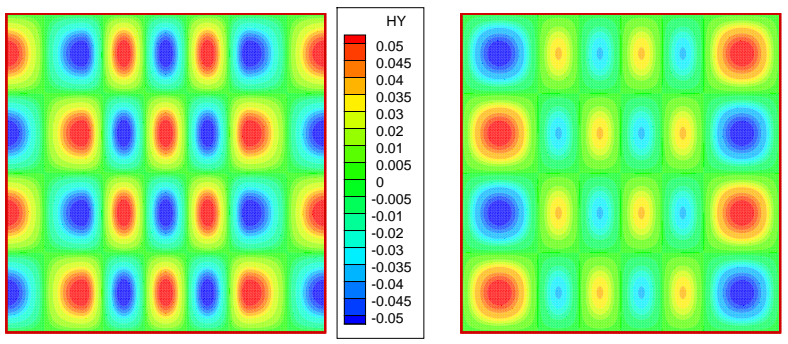

Fig. 3. Eigenmode in a PEC cavity containing a lossless material. Contour lines of $H_{y}$ (left) and $E_{z}$ (right) at $t=1.0$.

TABLE VI

NuMERICAL CONVERGENCE OF THE DGTD- $\mathbb{P}_{p}$ METHOD.

Conforming triangular mesh: convergence order.

\begin{tabular}{c|c|c|c|c}
\hline Interpolation order $p$ & $\mathbf{0}$ & $\mathbf{1}$ & $\mathbf{2}$ & $\mathbf{3}$ \\
\hline \hline Convergence order & 0.81 & 0.85 & 0.44 & 0.37 \\
\hline
\end{tabular}

Non-conforming triangular mesh: convergence order.

\begin{tabular}{c|c|c|c|c}
\hline Interpolation order $p$ & $\mathbf{0}$ & $\mathbf{1}$ & $\mathbf{2}$ & $\mathbf{3}$ \\
\hline \hline Convergence order & 0.85 & 0.82 & 0.36 & 0.34 \\
\hline
\end{tabular}

Tab. VIII shows the CPU times, the number of dof and the number of time steps to achieve a prescribed error level. The
TABLE VII

Numerical CONVERGENCE OF THE DGTD- $\mathbb{P}_{p_{c}}: \mathbb{P}_{p_{f}}$ METHOD.

Non-conforming triangular mesh: convergence order.

\begin{tabular}{c|c|c|c|c|c}
\hline Interpolation orders $p_{c}: p_{f}$ & $\mathbf{1 : 0}$ & $\mathbf{2 : 0}$ & $\mathbf{3 : 0}$ & $\mathbf{2 : 1}$ & $\mathbf{3 : 1}$ \\
\hline \hline Convergence order & 0.95 & 1.03 & 0.91 & 0.90 & 0.98 \\
\hline
\end{tabular}

results of the DGTD- $\mathbb{P}_{p_{c}}: \mathbb{P}_{p_{f}}$ on non-conforming meshes are very satisfactory comparing with the conforming DGTD- $\mathbb{P}_{p}$ method.

TABLE VIII

CPU TIME (SECONDS), NUMBER OF DOF AND NUMBER OF TIME STEPS TO ACHIEVE AN ERROR OF 3.0E-03 AT $t=1.0$

\begin{tabular}{c|c|c|c|c}
\multicolumn{5}{c}{ DGTD- $\mathbb{P}_{p}:$ Conforming triangular mesh. } \\
\hline$p$ & $\mathbf{0}$ & $\mathbf{1}$ & $\mathbf{2}$ & $\mathbf{3}$ \\
\hline$\#$ dof & 352800 & 194400 & 76800 & 32000 \\
$\# \Delta t$, CPU & $194, \quad 290$ & $276, \quad 110$ & $246, \quad 41$ & $184, \quad 12$ \\
\hline \multicolumn{5}{|c}{} \\
DGTD- $\mathbb{P}_{p_{c}}: \mathbb{P}_{p_{f}}:$ Non-conforming triangular mesh. \\
\hline$p_{c}: p_{f}$ & $\mathbf{2 : 0}$ & $\mathbf{2 : 1}$ & $\mathbf{3 : 1}$ & $\mathbf{3 : 2}$ \\
\hline \# dof & 86016 & 180000 & 140800 & 54400 \\
$\# \Delta t$, CPU & $368, \quad 102$ & 307,130 & 295,96 & $246, \quad 29$ \\
\hline
\end{tabular}

\section{CONCLUding REMARKS AND FUtURE WORKS}

We have presented preliminary results concerning a nonconforming discontinuous Galerkin method designed on unstructured triangular meshes for solving the time-domain Maxwell equations. The non-conformity is linked either to the use of locally refined meshes with an arbitrary-level of hanging nodes (i.e. $h$-refinement), or to the use of a space varying interpolation order (i.e. $p$-enrichment), or a combination of both (i.e. $h p$-refinement). Ongoing works target the extension to the numerical resolution of the 3D time-domain Maxwell equations considering unstructured tetrahedral meshes.

\section{REFERENCES}

[1] K. Yee, "Numerical solution of initial boundary value problems involving Maxwell's equations in isotropic media," IEEE Trans. Antennas and Propagat., vol. 14, no. 3, pp. 302-307, 1966.

[2] F. Collino, T. Fouquet, and P. Joly, "Conservative space-time mesh refinement methods for the FDTD solution of Maxwell's equations," $J$. Comp. Phys., vol. 211, no. 1, pp. 9-35, 2006.

[3] J. C. Nedelec, "A new family of mixed finite elements in $\mathbb{R}^{3}$," Numer. Math., vol. 50, pp. 57-81, 1986.

[4] A. Bossavit, "Solving Maxwell equations in a closed cavity, and the question of spurious modes," IEEE Trans. Magnetics, vol. 26, pp. 702$705,1990$.

[5] B. Cockburn, G. Karniadakis, and C. Shu, Eds., Discontinuous Galerkin methods. Theory, computation and applications, ser. Lecture Notes in Computational Science and Engineering. Springer-Verlag, 2000, vol. 11.

[6] G. Cohen, X. Ferrieres, and S. Pernet, "A spatial high spatial order hexahedral discontinuous Galerkin method to solve Maxwell's equations in time domain," J. Comp. Phys., vol. 217, no. 2, pp. 340-363, 2006.

[7] L. Fezoui, S. Lanteri, S. Lohrengel, and S. Piperno, "Convergence and stability of a discontinuous Galerkin time-domain method for the heterogeneous Maxwell equations on unstructured meshes," ESAIM: Math. Model. and Numer. Anal., vol. 39, no. 6, pp. 1149-1176, 2006.

[8] H. Fahs, S. Lanteri, and F. Rapetti, "A $h p$-like discontinuous Galerkin method for solving the 2D time-domain Maxwell's equations on nonconforming locally refined triangular meshes," INRIA, Research Reports 6023 and 6162, 2007, available: http://hal.inria.fr/inria-00140783/.

[9] S. Zhao and G. W. Wei, "High-order FDTD methods via derivative matching for Maxwell's equations with material interfaces," J. Comp. Phys., vol. 200, no. 1, pp. 60-103, 2004. 